\title{
Local distinguishability based genuinely quantum nonlocality without entanglement
}

\author{
Mao-Sheng Li, ${ }^{1,2}$ Yan-Ling Wang, ${ }^{3, *}$ Fei Shi, ${ }^{4, \dagger}$ and Man-Hong Yung ${ }^{1,5, \ddagger}$ \\ ${ }^{1}$ Department of Physics, Southern University of Science and Technology, Shenzhen, 518055, China \\ ${ }^{2}$ Department of Physics, University of Science and Technology of China, Hefei, 230026, China \\ ${ }^{3}$ School of Computer Science and Techonology, Dongguan University of Technology, Dongguan, 523808, China \\ ${ }^{4}$ School of Cyber Security, University of Science and Technology of China, Hefei, 230026, China. \\ ${ }^{5}$ Institute for Quantum Science and Engineering, and Department of Physics, \\ Southern University of Science and Technology, Shenzhen, 518055, China
}

\begin{abstract}
Recently, Halder et al. [Phys. Rev. Lett. 122, 040403 (2019)] proposed the concept strong nonlocality without entanglement: an orthogonal set of fully product states in multipartite quantum systems that is locally irreducible for every bipartition of the subsystems. As the difficulty of the problem, most of the results are restricted to tripartite systems. Here we consider a weaker form of nonlocality called local distinguishability based genuine nonlocality. A set of orthogonal multipartite quantum states is said to be genuinely nonlocal if it is locally indistinguishable for every bipartition of the subsystems. In this work, we tend to study the latter form of nonlocality. First, we present an elegant set of product states in bipartite systems that is locally indistinguishable. After that, based on a simple observation, we present a general method to construct genuinely nonlocal sets of multipartite product states by using those sets that are genuinely nonlocal but with less parties. As a consequence, we obtain that genuinely nonlocal sets of fully product states exist for all possible multipartite quantum systems.
\end{abstract}

\section{INTRODUCTION}

Quantum states discrimination plays a fundamental role in quantum information processing. It is well known that a set of quantum states can be perfectly distinguished by positive operation value measurement (POVM) if and only if these states are pairwise orthogonal [1]. In a multipartite setting, due to the physical obstacles, sometimes we can not take a global measurement but only can use local operations with classical communication (LOCC). Bennett et al. [2] presented examples of orthogonal product states that are indistinguishable under LOCC and named such a phenomenon as quantum nonlocality without entanglement. The nonlocality here is in the sense that there exists some quantum information that could be inferred from global measurement but cannot be read from local correlations of the subsystems. A set of orthogonal states which is indistinguishable under LOCC is also called as being locally indistinguishable or nonlocal. The local indistinguishability has been practically applied in quantum cryptography primitives such as data hiding $[3,4]$ and secret sharing [5-7].

Since the work of Bennett et al. [2], the problem of local discrimination of quantum states has attracted much

\footnotetext{
* wangylmath@yahoo.com

$\dagger$ shifei@mail.ustc.edu.cn

‡ yung@sustc.edu.cn
}

attention. The maximally entangled states and the product states, as being two extreme sets among the pure states, their local distinguishability is the most attractive. Here we present an incomplete list of the results about the local distinguishability of maximally entangled states [8-22] and product states [2, 23-43]. Another direction of related research is to study how much resource of entanglement are needed in order to distinguish quantum states which are locally indistinguishable [44-48]. Another important sets which are known to be locally indistinguishable are those unextendible product bases (UPB), sets of incomplete orthonormal product states whose complementary space has no product states [25, 26, 49-51].

Recently, Halder et al. [52] introduced a stronger form of local indistinguishability, i.e., local irreducibility. A set of multipartite orthogonal quantum states is said to be locally irreducible if it is not possible to locally eliminate one or more states from the set while preserving orthogonality of the postmeasurement states. Under this setting, they proposed the concept strong nonlocality without entanglement. A set of orthogonal multipartite product states is called to be strong nonlocality if it is locally irreducible for every bipartition of the systems. They provided the first two examples of strongly nonlocal sets of product states in $\mathbb{C}^{3} \otimes \mathbb{C}^{3} \otimes \mathbb{C}^{3}$ and $\mathbb{C}^{4} \otimes \mathbb{C}^{4} \otimes \mathbb{C}^{4}$ and raised the questions of how to extend their results to multipartite quantum systems and sets of unextendible product bases [52]. Quite recently, Zhang et al. [53] extended the concept of strong nonlocality to more general settings. However, there are only a few sets which have 
been proven to be strongly nonlocal [52-57]. Most of the known results are in the tripartite quantum settings. Here we propose a form of nonlocality called genuine nonlocality whose nonlocality is lying between the local distinguishablity based nonlocality and the local irreducibility based strong nonlocality (the definition here is slightly different from that defined by Rout et al. in Ref. [54]). A set of orthogonal multipartite quantum states is said to be genuinely nonlocal if it is locally indistinguishable for every bipartition of the systems. A nature question arises: are there genuinely nonlocal set of fully product states for any possible multipartite quantum systems? In this paper, we tend to address this problem.

The rest of this article is organized as follows. In Sec. II, we give some necessary notation, definitions and some basic result of local nonlocality of bipartite product basis. In Sec. III, we present a general method to obtaining genuinely nonlocal set of multipartite product states. Finally, we draw a conclusion and present some interesting problems in section IV.

\section{LOCALLY INDISTINGUISHABLE SET OF BIPARTITE PRODUCT STATES}

For any integer $n \geq 2$, we denote $U(n)$ to be the set of all unitary matrices of dimensional $n$. And throughout this paper, we use the following subset of unitary matrices

$$
U_{F L}(n):=\left\{\left(h_{i j}\right)_{i, j}^{n} \in U(n) \mid h_{1 k}, h_{k 1} \neq 0, k=1, \cdots, n\right\} .
$$

That is, the set of $n$ dimensional unitary matrices whose elements on the first and last rows are all nonzero.

Let $n \geq 3$ be an integer and $\mathcal{H}$ be a Hilbert space of dimensional $n$. Assume that $\mathcal{B}=(|1\rangle, \cdots,|n\rangle)$ is an $n$-tuple of vectors in $\mathcal{H}$ and these $n$ vectors are consisting of an orthonormal basis of $\mathcal{H}$. And we call $\mathcal{B}$ an ordered orthonormal basis of $\mathcal{H}$. For any $H=\left(h_{j k}\right)_{j, k=1}^{n-1} \in U_{F L}(n-1)$, we define two operations on $\mathcal{H}$ with respect to $\mathcal{B}$

$$
H_{\mathcal{B}}^{(U)}:=\sum_{j=1}^{n-1} \sum_{k=1}^{n-1} h_{j k}|j\rangle\left\langle k\left|, H_{\mathcal{B}}^{(D)}:=\sum_{j=1}^{n-1} \sum_{k=1}^{n-1} h_{j k}\right| j+1\right\rangle\langle k+1| .
$$

That is, under the computational basis $\{|1\rangle, \cdots,|n\rangle\}$, their matrix representations are as follows

$$
\begin{aligned}
& H_{\mathcal{B}}^{(U)}=\left[\begin{array}{ll}
H & \mathbf{0}_{(n-1) \times 1} \\
\mathbf{0}_{1 \times(n-1)} & 0
\end{array}\right], \\
& H_{\mathcal{B}}^{(D)}=\left[\begin{array}{ll}
0 & \mathbf{0}_{1 \times(n-1)} \\
\mathbf{0}_{(n-1) \times 1} & H
\end{array}\right] .
\end{aligned}
$$

We call them the up extension and down extension of $H$ with respect to $\mathcal{B}$ respectively.

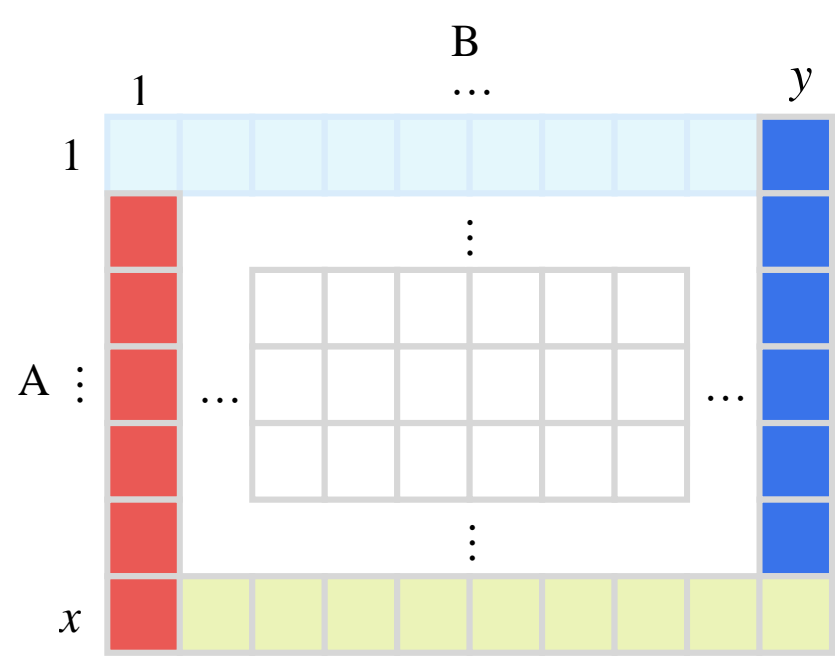

FIG. 1. States structure corresponding to $\{|\psi\rangle\}$ in Theorem 1 (or $\{|\Psi\rangle\}$ in Theorem 3).

Motivated by the constructions of nonlocal sets of product states in Ref. [42], the following theorem is a generalized version of their results but the proof is more elegant.

Theorem 1 Let $x, y \geq 3$ be integers and $X \in U_{F L}(x-1)$, $Y \in U_{F L}(y-1)$. Let $\mathcal{H}_{A}\left(\mathcal{H}_{B}\right)$ be a Hilbert space of $d i$ mension $x(y)$ with an ordered orthonormal basis $\mathcal{A}=$ $\left(|1\rangle_{A}, \cdots,|x\rangle_{A}\right)\left(\mathcal{B}=\left(|1\rangle_{B}, \cdots,|y\rangle_{B}\right)\right)$. The following $2(x+y)-4$ product states in $\mathcal{H}_{A} \otimes \mathcal{H}_{B}$ are locally indistinguishable (See Fig. 1)

$$
\begin{aligned}
& \left|\psi_{i}\right\rangle:=|1\rangle_{A} \otimes\left(Y_{\mathcal{B}}^{(U)}|i\rangle_{B}\right), \\
& \left|\psi_{y-1+j}\right\rangle:=\left(X_{\mathcal{A}}^{(U)}|j\rangle_{A}\right) \otimes|y\rangle_{B}, \\
& \left|\psi_{x+y-3+k}\right\rangle:=|x\rangle_{A} \otimes\left(Y_{\mathcal{B}}^{(D)}|k\rangle_{B}\right), \\
& \left|\psi_{x+2 y-4+l}\right\rangle:=\left(X_{\mathcal{A}}^{(D)}|l\rangle_{A}\right) \otimes|1\rangle_{B},
\end{aligned}
$$

where $1 \leq i \leq y-1,1 \leq j \leq x-1,2 \leq k \leq y, 2 \leq l \leq x$.

Proof. Suppose Alice starts with a measurement $\left\{M_{a}^{\dagger} M_{a}\right\}_{a=1}^{S}$. The postmeasurement states should be orthogonal to each other, i.e.

$$
\left\langle\psi_{i}\left|M_{a}^{\dagger} M_{a} \otimes \mathbb{I}_{B}\right| \psi_{j}\right\rangle=0, \text { for } i \neq j
$$

Let $M:=M_{a}^{\dagger} M_{a}$. Suppose its matrix representation under the ordered basis $\mathcal{A}$ is $\left(m_{i j}\right)_{i, j=1}^{x}$. Then one finds

$$
M=\sum_{i=1}^{x} \sum_{j=1}^{x} m_{i j}|i\rangle_{A}\langle j|
$$



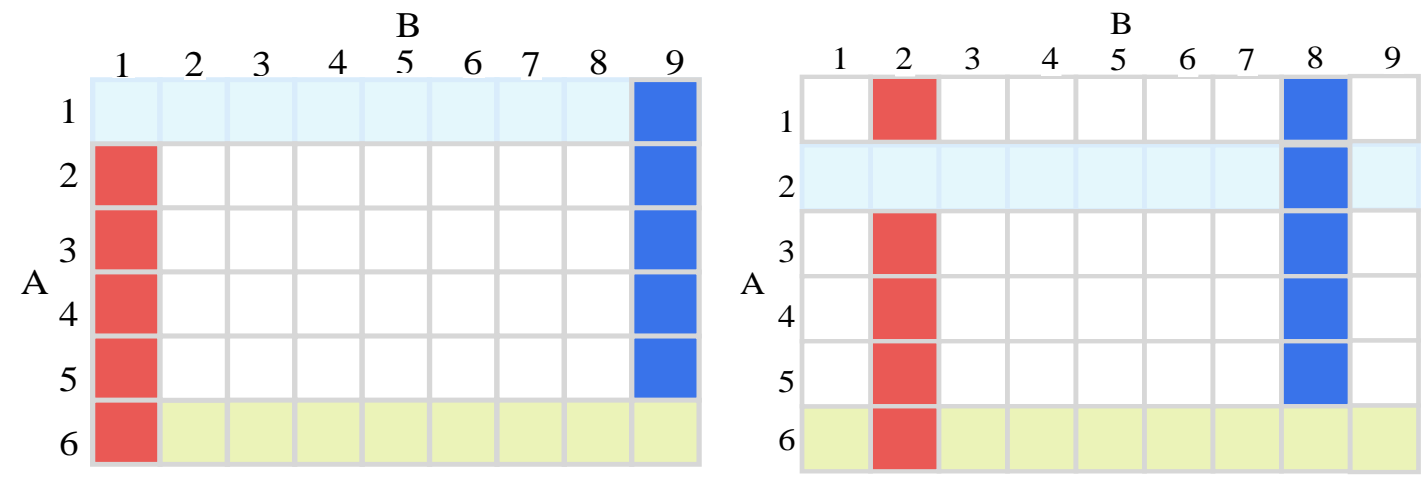

FIG. 2. The left hand side draw the boundary states corresponding to the ordered bases $\mathcal{A}:=\left(|1\rangle_{A},|2\rangle_{A},|3\rangle_{A},|4\rangle_{A},|5\rangle_{A},|6\rangle_{A}\right)$ and $\mathcal{B}:=\left(|1\rangle_{B},|2\rangle_{B},|3\rangle_{B},|4\rangle_{B},|5\rangle_{B},|6\rangle_{B},|7\rangle_{B},|8\rangle_{B},|9\rangle_{B}\right)$. The right hand side presents the boundary states corresponding to the ordered bases $\mathcal{A}^{\prime}:=\left(|2\rangle_{A},|1\rangle_{A},|3\rangle_{A},|4\rangle_{A},|5\rangle_{A},|6\rangle_{A}\right)$ and $\mathcal{B}^{\prime}:=\left(|2\rangle_{B},|1\rangle_{B},|3\rangle_{B},|4\rangle_{B},|5\rangle_{B},|6\rangle_{B},|7\rangle_{B},|9\rangle_{B},|8\rangle_{B}\right)$

but draws them under the ordered bases $\mathcal{A}$ and $\mathcal{B}$.

Because $M_{a} \otimes \mathbb{I}_{y}\left|\psi_{1}\right\rangle$ is orthogonal to the set of states $\left\{M_{a} \otimes \mathbb{I}_{y}\left|\psi_{x+2 y-4+l}\right\rangle \mid l=2,3, \cdots, x\right\}$, we have the following equations

$$
{ }_{A}\left\langle 1\left|M X_{\mathcal{A}}^{(D)}\right| l\right\rangle_{A}=0, l=2,3, \cdots, x .
$$

These equalities are equivalent to the matrix equality $\left[m_{12}, m_{13}, \cdots, m_{1 x}\right] X=[0,0, \cdots, 0]$. As $X$ is invertible, we have $\left[m_{12}, m_{13}, \cdots, m_{1 x}\right]=[0,0, \cdots, 0]$. As $M$ is Hermitian, we also have $\left[m_{21}, m_{31}, \cdots, m_{x 1}\right]=[0,0, \cdots, 0]$.

Because $M_{a} \otimes \mathbb{I}_{y}\left|\psi_{x+y-3+2}\right\rangle$ is orthogonal to the set of states $\left\{M_{a} \otimes \mathbb{I}_{y}\left|\psi_{y-1+j}\right\rangle \mid j=1,2, \cdots, x-1\right\}$, we have the following equations

$$
{ }_{A}\left\langle x\left|M X_{\mathcal{A}}^{(U)}\right| j\right\rangle_{A}=0, j=1,2, \cdots, y-1 .
$$

These equalities are equivalent to the matrix equality $\left[m_{x 1}, m_{x 2}, \cdots, m_{x(x-1)}\right] X=[0,0, \cdots, 0]$. As $X$ is invertible, we have $\left[m_{x 1}, m_{x 2}, \cdots, m_{x(x-1)}\right]=[0,0, \cdots, 0]$. As $M$ is Hermitian, we also have $\left[m_{1 x}, m_{2 x}, \cdots, m_{(x-1) x}\right]=$ $[0,0, \cdots, 0]$.

Because the set of states $\left\{M_{a} \otimes \mathbb{I}_{y}\left|\psi_{y-1+j}\right\rangle \mid j=\right.$ $1,2, \cdots, x-1\}$ are pairwise orthogonal to each other, we have the following equations

$$
{ }_{A}\left\langle j_{1}\left|X_{\mathcal{A}}^{(U)^{\dagger}} M X_{\mathcal{A}}^{(U)}\right| j_{2}\right\rangle_{A}=0, \text { for } 1 \leq j_{1} \neq j_{2} \leq x-1
$$

If we define $M^{(u)}:=\left(m_{i j}\right)_{i, j=1}^{x-1}$, the above equalities are equivalent to $X^{\dagger} M^{(u)} X=\operatorname{diag}\left(\alpha_{1}, \cdots, \alpha_{x-1}\right)$. Since $X$ is a unitary matrix, we have

$$
M^{(u)} X=X \operatorname{diag}\left(\alpha_{1}, \cdots, \alpha_{x-1}\right) .
$$

In the following, we will compare the first row of the matrices at both hand sides of the above equality. Suppose that $X=\left(X_{i j}\right)_{i, j=1}^{x-1}$. Then the first row of $M^{(u)} X$ is $\left[m_{11} X_{11}, m_{11} X_{12}, \cdots, m_{11} X_{1(x-1)}\right]$. Meanwhile, the first row of $X \operatorname{diag}\left(\alpha_{1}, \cdots, \alpha_{x-1}\right)$ is $\left[\alpha_{1} X_{11}, \alpha_{2} X_{12}, \cdots, \alpha_{x-1} X_{1(x-1)}\right]$. Comparing these two vectors, one can derive $\left[\alpha_{1}, \alpha_{2}, \cdots, \alpha_{x-1}\right]=$ $\left[m_{11}, m_{11}, \cdots, m_{11}\right]$ as $X_{11}, X_{12}, \cdots, X_{1(x-1)}$ are all nonzero. Hence

$$
M^{(u)}=X \operatorname{diag}\left(\alpha_{1}, \cdots, \alpha_{x-1}\right) X^{\dagger}=m_{11} \mathbb{I}_{x-1} .
$$

Using the orthogonal relations among the states in $\left\{M_{a} \otimes \mathbb{I}_{y}\left|\psi_{x+2 y-4+l}\right\rangle \mid l=2,3, \cdots, x\right\}$, we have

$$
{ }_{A}\left\langle l_{1}\left|X_{\mathcal{A}}^{(D)}{ }^{\dagger} M X_{\mathcal{A}}^{(D)}\right| l_{2}\right\rangle_{A}=0, \text { for } 2 \leq l_{1} \neq l_{2} \leq x .
$$

If we define $M^{(d)}:=\left(m_{i j}\right)_{i, j=2}^{x}$, the above equalities are equivalent to $X^{\dagger} M^{(d)} X=\operatorname{diag}\left(\beta_{2}, \cdots, \beta_{x}\right)$. In a similar way (but here we should consider the last row instead of the first row), we can get $M^{(d)}=m_{x x} \mathbb{I}_{x-1}$.

Therefore, the Hermitian matrix $M$ is of the form $m_{11} \mathbb{I}_{x}$. That is, Alice can only start with trivial measurement. By the symmetry of the constructed states, Bob can only start with trivial measurement.

Remark: Notice that the product states we constructed in Theorem 1 are spanned by the "boundary states" with respect to the ordered bases $\mathcal{A}$ and $\mathcal{B}$ (the outermost layer of a rectangle under the ordered bases $\mathcal{A}$ and $\mathcal{B}$ ). One finds that $\operatorname{span}_{\mathbb{C}}\left\{\left|\psi_{1}\right\rangle, \cdots,\left|\psi_{2 x+2 y-4}\right\rangle\right\}$ is equal to

$$
\operatorname{span}_{\mathbb{C}}\left\{\mathcal{A}_{i} \otimes \mathcal{B}_{j} \mid i \in\{1, x\} \text { or } j \in\{1, y\}\right\}
$$

where $\mathcal{A}_{i}\left(\mathcal{B}_{j}\right)$ is the $i$-th $(j$-th) element of the $x(y)$-tuples $\mathcal{A}(\mathcal{B})$ (see Fig. 2). 


\section{CONSTRUCTING GENUINELY NONLOCAL SET FROM KNOWN ONES}

As any set of orthogonal product states in $\mathbb{C}^{2} \otimes \mathbb{C}^{d}$ is locally distinguishable [26], a necessary condition for an orthogonal set of fully product states in $\bigotimes_{i=1}^{L} \mathbb{C}^{d_{i}}$ to be genuinely nonlocal is $d_{i} \geq 3$ for all $i$. In this section, we show that there always exists some genuinely nonlocal set of fully product states in $\bigotimes_{i=1}^{L} \mathbb{C}^{d_{i}}$ if the previous necessary condition is fulfilled.

Theorem 2 Let $L \geq 3$ and $d_{i} \geq 3(i=1,2, \cdots, L)$ be integers. Then there always exists an orthogonal set of fully product states in $\otimes_{i=1}^{L} \mathbb{C}^{d_{i}}$ that is genuinely nonlocal.

This conclusion can be derived from Theorem 1, Theorem 3, Proposition 1 of this paper and the genuinely nonlocal set of $\mathbb{C}^{3} \otimes \mathbb{C}^{3} \otimes \mathbb{C}^{3}$ constructed in Ref. [54].

Notice that if $S=\left\{\left|\phi_{k}\right\rangle_{A}\left|\theta_{k}\right\rangle_{B}\right\}_{k=1}^{N}$ is $A \mid B$ locally indistinguishable, then $\mathcal{S}=\left\{\left|\phi_{k}\right\rangle_{A}\left|\theta_{k}\right\rangle_{B}|\varphi\rangle_{A_{1}}|\vartheta\rangle_{B_{1}}\right\}_{k=1}^{N}$ is also $A A_{1} \mid B B_{1}$ locally indistinguishable. Otherwise, in the first setting, Alice and Bob can prepare the ancillar qudit states as $|\varphi\rangle_{A_{1}},|\vartheta\rangle_{B_{1}}$ respectively on themselves side and using the latter distinguish strategy to locally distinguish the set of states in $S$. Moreover, we have the following observation(see also in Ref. [55])

Observation 1 Let $S=\left\{\left|\Psi_{k}\right\rangle_{A B}\right\}_{k=1}^{N}$ be a nonlocal product set shared between Alice and Bob. Consider the set $\mathcal{S}:=\left\{\left|\Psi_{k}\right\rangle_{A B} \otimes\left|\Phi_{0}\right\rangle_{A_{1} \cdots A_{m}} \otimes\left|\Theta_{0}\right\rangle_{B_{1} \cdots B_{n}}\right\}_{k=1}^{N}$, where $\left|\Phi_{0}\right\rangle_{A_{1} \cdots A_{m}}$ and $\left|\Theta_{0}\right\rangle_{B_{1} \cdots B_{n}}$ are some fully product states with some of the subsystems $\left\{A_{i}\right\}_{i=1}^{m}$ and $\left\{B_{j}\right\}_{j=1}^{n}$ being in possession of some parties. The resulting set $\mathcal{S}$ is also nonlocal between $\mathcal{H}_{A} \otimes\left(\otimes_{i=1}^{m} \mathcal{H}_{A_{i}}\right)$ and $\mathcal{H}_{B} \otimes\left(\otimes_{j=1}^{n} \mathcal{H}_{B_{j}}\right)$.

With this observation, we show how to use the special structure of nonlocal sets in Theorem 1 to construct genuinely nonlocal set of product states in tripartite systems.

Theorem 3 Let $x, z \geq 3, y \geq 4$ be integers and $X, Y, Z$ belong to $U_{F L}(x-1), \bar{U}_{F L}(y-1)$ and $U_{F L}(z-1)$ respectively. Let $\mathcal{H}_{A}, \mathcal{H}_{B}, \mathcal{H}_{C}$ be Hilbert spaces of dimension $x, y, z$ respectively. Suppose that $\mathcal{A}=\left(|1\rangle_{A}, \cdots,|x\rangle_{A}\right)$, $\mathcal{B}=\left(|1\rangle_{B}, \cdots,|y\rangle_{B}\right)$, and $\mathcal{C}=\left(|1\rangle_{C}, \cdots,|z\rangle_{C}\right)$ are ordered orthonormal bases with respect to $\mathcal{H}_{A}, \mathcal{H}_{B}, \mathcal{H}_{C}$. The following $2 x+4 y+2 z-8$ product states in $\mathbb{C}^{x} \otimes$ $\mathbb{C}^{y} \otimes \mathbb{C}^{z}$ are pairwise orthogonal and they form a set of product states which is genuinely nonlocal (see Fig. 1)

$$
\begin{aligned}
& \left|\Psi_{i}\right\rangle:=|1\rangle_{A} \otimes\left(Y_{\mathcal{B}}^{(U)}|i\rangle_{B}\right) \otimes|1\rangle_{C}, \\
& \left|\Psi_{y-1+j}\right\rangle:=\left(X_{\mathcal{A}}^{(U)}|j\rangle_{A}\right) \otimes|y\rangle_{B} \otimes|1\rangle_{C}, \\
& \left|\Psi_{x+y-3+k}\right\rangle:=|x\rangle_{A} \otimes\left(Y_{\mathcal{B}}^{(D)}|k\rangle_{B}\right) \otimes|1\rangle_{C}, \\
& \left|\Psi_{x+2 y-4+l}\right\rangle:=\left(X_{\mathcal{A}}^{(D)}|l\rangle_{A}\right) \otimes|1\rangle_{B} \otimes|1\rangle_{C},
\end{aligned}
$$

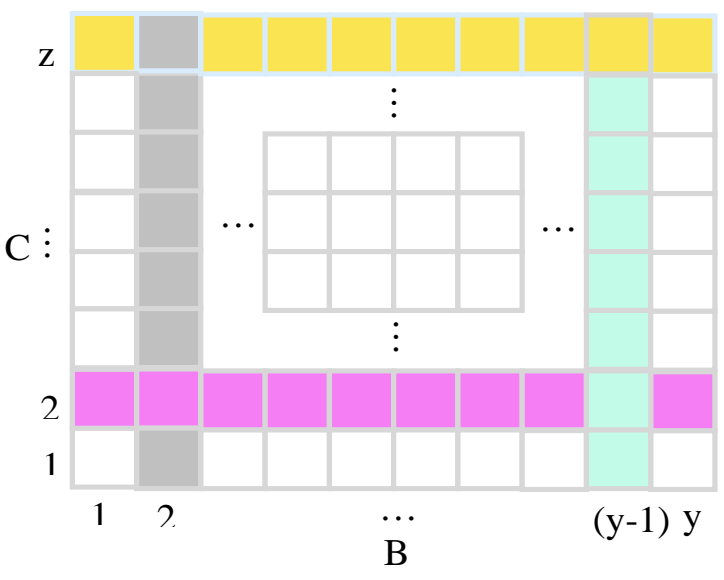

FIG. 3. States structure corresponding to $\{|\Phi\rangle\}$ in the Theorem 3 .

where $1 \leq i \leq y-1,1 \leq j \leq x-1,2 \leq k \leq y, 2 \leq l \leq x$ and (see Fig. 3)

$$
\begin{aligned}
& \left|\Phi_{i}\right\rangle:=|2\rangle_{A} \otimes\left|1^{\prime}\right\rangle_{B} \otimes\left(Z_{\mathcal{C}^{\prime}}^{(U)}\left|i^{\prime}\right\rangle_{C}\right), \\
& \left|\Phi_{z-1+j}\right\rangle:=|2\rangle_{A} \otimes\left(Y_{\mathcal{B}^{\prime}}^{(U)}\right)\left|j^{\prime}\right\rangle_{B} \otimes\left|z^{\prime}\right\rangle_{C}, \\
& \left|\Phi_{y+z-3+k}\right\rangle:=|2\rangle_{A} \otimes\left|y^{\prime}\right\rangle_{B} \otimes\left(Z_{\mathcal{C}^{\prime}}^{(D)}\left|k^{\prime}\right\rangle_{C}\right), \\
& \left|\Phi_{y+2 z-4+l}\right\rangle:=|2\rangle_{A} \otimes\left(Y_{\mathcal{B}^{\prime}}^{(D)}\left|l^{\prime}\right\rangle_{B}\right) \otimes\left|1^{\prime}\right\rangle_{C} .
\end{aligned}
$$

where $1 \leq i \leq z-1,1 \leq j \leq y-1,2 \leq k \leq z, 2 \leq l \leq y$. Here the ordered bases $\mathcal{B}^{\prime}$ and $\mathcal{C}^{\prime}$ are defined as follows

$$
\begin{aligned}
& \mathcal{B}^{\prime}:=\left(\left|1^{\prime}\right\rangle_{B},\left|2^{\prime}\right\rangle_{B}, \cdots,\left|(y-1)^{\prime}\right\rangle_{B},\left|y^{\prime}\right\rangle_{B}\right), \\
& \mathcal{C}^{\prime}:=\left(\left|1^{\prime}\right\rangle_{C},\left|2^{\prime}\right\rangle_{C}, \cdots,\left|z^{\prime}\right\rangle_{C}\right)
\end{aligned}
$$

where $\left|1^{\prime}\right\rangle_{B}=|2\rangle_{B},\left|2^{\prime}\right\rangle_{B}=|1\rangle_{B},\left|y-1^{\prime}\right\rangle_{B}=|y\rangle_{B}$, $\left|y^{\prime}\right\rangle_{B}=|y-1\rangle_{B},\left|j^{\prime}\right\rangle_{B}=|j\rangle_{B}$ for $3 \leq j \leq y-1$ and $\left|1^{\prime}\right\rangle_{C}=|2\rangle_{C},\left|2^{\prime}\right\rangle_{C}=|1\rangle_{C},\left|k^{\prime}\right\rangle_{C}=|k\rangle_{C}$ for $3 \leq j \leq z$.

Proof. We notice that $\operatorname{span}_{\mathbb{C}}\left\{\left|\Psi_{1}\right\rangle,\left|\Psi_{2}\right\rangle \cdots,\left|\Psi_{2 x+2 y-4}\right\rangle\right\}$ is equal to the linear space spanned by

$$
\mathcal{S}_{\Psi}:=\left\{|i\rangle_{A}|j\rangle_{B}|1\rangle_{C} \mid \quad i \in\{1, x\} \text { or } j \in\{1, y\}\right\}
$$

while $\operatorname{span}_{\mathbb{C}}\left\{\left|\Phi_{1}\right\rangle,\left|\Phi_{2}\right\rangle, \cdots,\left|\Phi_{2 y+2 z-4}\right\rangle\right\}$ is equal to the linear space spanned by

$$
\begin{aligned}
& \mathcal{S}_{\Phi}:=\left\{|2\rangle_{A}\left|j^{\prime}\right\rangle_{B}\left|k^{\prime}\right\rangle_{C} \mid j \in\{1, y\} \text { or } k \in\{1, z\}\right\} \\
& =\left\{|2\rangle_{A}|j\rangle_{B}|k\rangle_{C} \mid j \in\{2, y-1\} \text { or } k \in\{2, z\}\right\} .
\end{aligned}
$$

As $\mathcal{S}_{\Psi} \cap \mathcal{S}_{\Phi}=\emptyset$ and $\mathcal{S}_{\Psi}, \mathcal{S}_{\Phi} \subseteq\left\{|i\rangle_{A}|j\rangle_{B}|k\rangle_{C} \mid 1 \leq i \leq\right.$ $x, 1 \leq j \leq y, 1 \leq k \leq z\}$ which is a orthonormal basis of $\mathcal{H}_{A} \otimes \mathcal{H}_{B} \otimes \mathcal{H}_{C}$, we have $\left\langle\Psi_{u} \mid \Phi_{v}\right\rangle=0$ for integers $u, v$ with $1 \leq u \leq 2 x+2 y-4,1 \leq v \leq 2 y+2 z-4$. 


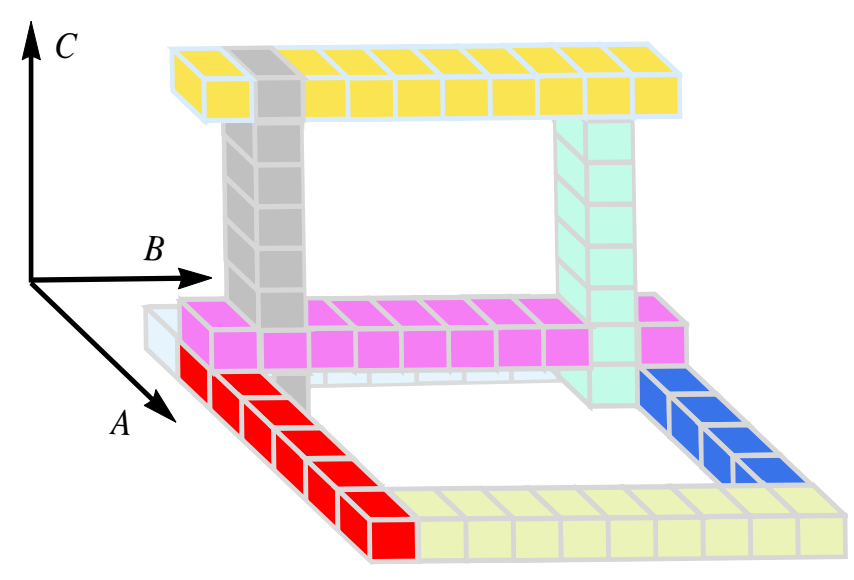

FIG. 4. This is a schematic diagram for the states in Theorem 3.

Therefore, the states in $\left\{\left|\Psi_{u}\right\rangle\right\}_{u=1}^{2 x+2 y-4} \cup\left\{\left|\Phi_{v}\right\rangle\right\}_{v=1}^{2 y+2 z-4}$ are pairwise orthogonal ( Fig. 4 is more intuitive for the orthogonality).

To prove that the set of states we construct are genuinely nonlocal. There are only three ways to separate $A B C$ into two sets. That is, $A|B C, B| C A, C \mid A B$. By Theorem 1 and Observation 1 , the set $\left\{\left|\Psi_{u}\right\rangle\right\}_{u=1}^{2 x+2 y-4}$ is locally indistinguishable as the partitions $A \mid B C$ and $B \mid C A$. And the set $\left\{\left|\Phi_{v}\right\rangle\right\}_{v=1}^{2 y+2 z-4}$ is locally indistinguishable as the partitions $B \mid C A$ and $C \mid A B$. Hence the given set is genuinely nonlocal.

In the following, we begin to strengthen the results in [37] where they constructed locally indistinguishable multipartite product states from known bipartite ones. The following two propositions enhance their results to genuine nonlocality settings.

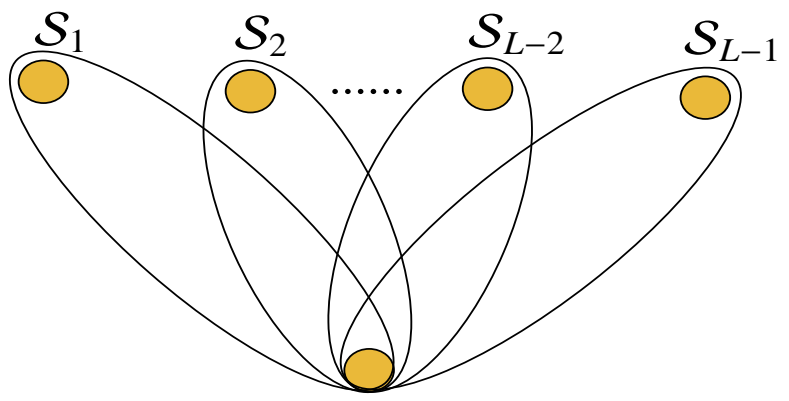

FIG. 5. States structure corresponding to the Proposition 1.
Proposition 1 Let $L \geq 4$ be an integer and $d_{i} \geq 3$ for all $1 \leq i \leq L$. Let $S_{i}=\left\{\left|\psi_{j}^{(i)}\right\rangle\left|\phi_{j}^{(i)}\right\rangle\right\}_{j=1}^{n_{i}} \subseteq \mathbb{C}^{d_{1}} \otimes \mathbb{C}^{d_{i+1}}$ be sets of product states that are locally indistinguishable for $i=1,2, \cdots, L-1$. Then the union of the following sets (See Fig. 5)

$$
\begin{gathered}
\mathcal{S}_{1}=\left\{\left|\psi_{j}^{(1)}\right\rangle\left|\phi_{j}^{(1)}\right\rangle|1\rangle|1\rangle|1\rangle \cdots|2\rangle\right\}_{j=1}^{n_{1}}, \\
\mathcal{S}_{2}=\left\{\left|\psi_{j}^{(2)}\right\rangle|2\rangle\left|\phi_{j}^{(2)}\right\rangle|1\rangle|1\rangle \cdots|1\rangle\right\}_{j=1}^{n_{2}}, \\
\mathcal{S}_{3}=\left\{\left|\psi_{j}^{(3)}\right\rangle|1\rangle|2\rangle\left|\phi_{j}^{(3)}\right\rangle|1\rangle \cdots|1\rangle\right\}_{j=1}^{n_{3}}, \\
\vdots \\
\mathcal{S}_{L-1}=\left\{\left|\psi_{j}^{(L-1)}\right\rangle|1\rangle|1\rangle \cdots|1\rangle|2\rangle\left|\phi_{j}^{(L-1)}\right\rangle\right\}_{j=1}^{n_{L-1}}
\end{gathered}
$$

is also a genuinely nonlocal set of product states in $\mathbb{C}^{d_{1}} \otimes$ $\mathbb{C}^{d_{2}} \otimes \cdots \otimes \mathbb{C}^{d_{L}}$

Proof. To distinguish the states of $\mathcal{S}_{1}$, by Observation 1 , the first two parties must come together and perform a global measurement. Similarly, to distinguish the states of $\mathcal{S}_{i}$, the 1 -th and $(i+1)$-th parties must come together and perform a global measurement.

Therefore, all the parties must come together to distinguish all the states in Eq. (1). Hence such a set of states is genuinely nonlocal.

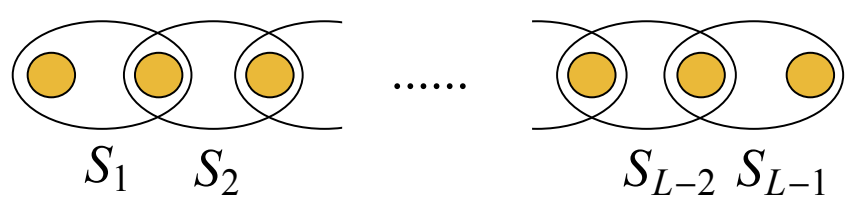

FIG. 6. States structure corresponding to the Proposition 2.

Proposition 2 Let $L \geq 5$ be an integer and $d_{i} \geq 3$ for all $1 \leq i \leq L$. Let $S_{i}:=\left\{\left|\psi_{j}^{(i)}\right\rangle\left|\phi_{j}^{(i)}\right\rangle\right\}_{j=1}^{n_{i}} \subseteq \mathbb{C}^{d_{i}} \otimes \mathbb{C}^{d_{i+1}}$ be sets of product states that are locally indistinguishable for $i=1,2, \cdots, L-1$. Then the union of the following sets (See Fig. 6)

$$
\begin{aligned}
& \mathcal{S}_{1}=\left\{\left|\psi_{j}^{(1)}\right\rangle\left|\phi_{j}^{(1)}\right\rangle|2\rangle|1\rangle|1\rangle|1\rangle \cdots|1\rangle\right\}_{j=1}^{n_{1}}, \\
& \mathcal{S}_{2}=\left\{|1\rangle\left|\psi_{j}^{(2)}\right\rangle\left|\phi_{j}^{(2)}\right\rangle|2\rangle|1\rangle|1\rangle \cdots|1\rangle\right\}_{j=1}^{n_{2}}, \\
& \mathcal{S}_{3}=\left\{|1\rangle|1\rangle\left|\psi_{j}^{(3)}\right\rangle\left|\phi_{j}^{(3)}\right\rangle|2\rangle|1\rangle \cdots|1\rangle\right\}_{j=1}^{n_{3}},
\end{aligned}
$$

$$
\mathcal{S}_{L-1}=\left\{|2\rangle|1\rangle|1\rangle|1\rangle \cdots|1\rangle\left|\psi_{j}^{(L-1)}\right\rangle\left|\phi_{j}^{(L-1)}\right\rangle\right\}_{j=1}^{n_{L-1}}
$$


is also a genuinely nonlocal set of product states in $\mathbb{C}^{d_{1}} \otimes$ $\mathbb{C}^{d_{2}} \otimes \cdots \otimes \mathbb{C}^{d_{L}}$.

To construct multipartite genuinely nonlocal sets, instead of using bipartite nonlocal product states, we can also start with some known genuinely nonlocal sets of product states in tripartite systems.

Proposition 3 Let $L \geq 3$ be an integer. Let $\left\{\left|\psi_{j}\right\rangle\left|\phi_{j}\right\rangle\left|\chi_{j}\right\rangle\right\}_{j=1}^{n} \subseteq \mathbb{C}^{3} \otimes \mathbb{C}^{3} \otimes \mathbb{C}^{3}$ be a set of product states that is genuinely nonlocal. Then the union of the following sets (See Fig. 7)

$$
\begin{gathered}
\mathcal{S}_{1}=\left\{\left|\psi_{j}\right\rangle\left|\phi_{j}\right\rangle\left|\chi_{j}\right\rangle|1\rangle|1\rangle|1\rangle \cdots|1\rangle|2\rangle|2\rangle\right\}_{j=1}^{n}, \\
\mathcal{S}_{2}=\left\{\left|\psi_{j}\right\rangle|2\rangle|2\rangle\left|\phi_{j}\right\rangle\left|\chi_{j}\right\rangle|1\rangle \cdots|1\rangle|1\rangle|1\rangle\right\}_{j=1}^{n}, \\
\mathcal{S}_{3}=\left\{\left|\psi_{j}\right\rangle|1\rangle|1\rangle|2\rangle|2\rangle\left|\phi_{j}\right\rangle\left|\chi_{j}\right\rangle|1\rangle \cdots|1\rangle\right\}_{j=1}^{n}, \\
\vdots \\
\mathcal{S}_{L}=\left\{\left|\psi_{j}\right\rangle|1\rangle|1\rangle|1\rangle|1\rangle \cdots|2\rangle|2\rangle\left|\phi_{j}\right\rangle\left|\chi_{j}\right\rangle\right\}_{j=1}^{n},
\end{gathered}
$$

is also a genuinely nonlocal set of product states in $\bigotimes_{i=1}^{2 L+1} \mathcal{H}_{A_{i}}$ where $\mathcal{H}_{A_{i}}=\mathbb{C}^{3}$.

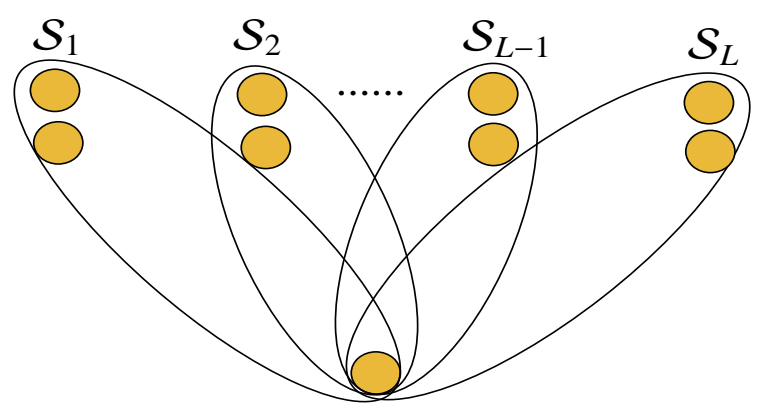

FIG. 7. States structure corresponding to the Proposition 3.

In fact, the above constructions can be extended to much more general settings. Let $L \geq 3$ be an integer and $\mathcal{P}:=\{1,2,3, \cdots, L\}$. Let $\mathcal{H}:=\otimes_{j \in \mathcal{P}} \mathcal{H}_{j}$ be an $L$-parties quantum system. Suppose there are $s$ proper subsets of $\mathcal{P}: \mathcal{P}_{1}, \mathcal{P}_{2}, \cdots, \mathcal{P}_{s}$ and we denote $\overline{\mathcal{P}}_{i}:=\mathcal{P} \backslash \mathcal{P}_{i}$ for each $i$. We make the following assumptions:

(a) $S_{i}=\left\{\left|\Psi_{j}\right\rangle_{\mathcal{P}_{i}}\right\}_{j=1}^{n_{i}}$ is a genuinely nonlocal product set in $\mathcal{H}_{\mathcal{P}_{i}}:=\otimes_{j \in \mathcal{P}_{i}} \mathcal{H}_{j}$ for each $i \in\{1,2, \cdots, s\}$.

(b) There is a fully product state $\left|\Phi_{i}\right\rangle_{\overline{\mathcal{P}}_{i}} \in \otimes_{j \in \overline{\mathcal{P}}_{i}} \mathcal{H}_{j}$ for each $i$ such that the states in the union of the sets $\mathcal{S}_{i}=\left\{\left|\Psi_{j}\right\rangle_{\mathcal{P}_{i}}\left|\Phi_{i}\right\rangle_{\overline{\mathcal{P}}_{i}}\right\}_{j=1}^{n_{i}}(1 \leq i \leq s)$ are mutually orthogonal to each other.
We use the notation $\mathfrak{P}:=\left(\mathcal{P},\left\{\mathcal{P}_{1}, \mathcal{P}_{2}, \cdots, \mathcal{P}_{s}\right\}\right)$. For each $\mathfrak{P}$, We attach it a graph $G_{\mathfrak{P}}=\left(V_{\mathfrak{P}}, E_{\mathfrak{P}}\right)$ defined as follows: its vertex set is $V_{\mathfrak{P}}=\mathcal{P}$ and its edge set is

$$
E_{\mathfrak{P}}=\bigcup_{i=1}^{s}\left\{\left(u_{i}, v_{i}\right) \mid u_{i}, v_{i} \in \mathcal{P}_{i} \text { and } u_{i} \neq v_{i}\right\} \text {. }
$$

Theorem 4 Under the notation and assumptions of the two paragraphs previous and suppose that $G_{\mathfrak{P}}$ is connected, then the set $\mathcal{S}:=\cup_{i=1}^{s} \mathcal{S}_{i}$ is a genuinely nonlocal set of product states in $\otimes_{j \in \mathcal{P}} \mathcal{H}_{j}$.

Proof. Suppose not, there exist a nontrivial bipartition of $\mathcal{P}$, say $U \mid V$ (both $U$ and $V$ are nonempty subset of $\mathcal{P}$ ), such that the set $\mathcal{S}$ is locally distinguishable when considering as a set of bipartite states in $\left(\otimes_{j \in U} \mathcal{H}_{j}\right) \otimes\left(\otimes_{j \in V} \mathcal{H}_{j}\right)$. As the connectivity of $G_{\mathfrak{P}}$, there must exist some edge $(u, v) \in E_{\mathfrak{P}}$ which connects the two sets $U$ and $V$, i.e. $u \in U$ and $v \in V$. By the definition of $E_{\mathfrak{P}}$, there exist some $i$ such that $u, v \in \mathcal{P}_{i}$. However, the set $S_{i}$ is genuinely nonlocal in $\mathcal{H}_{\mathcal{P}_{i}}$ by the assumption (a) above. So it is locally indistinguishable for the partition

$$
\left(U \cap \mathcal{P}_{i}\right) \mid\left(V \cap \mathcal{P}_{i}\right)
$$

of $\mathcal{P}_{i}$ as both $U \cap \mathcal{P}_{i}$ and $V \cap \mathcal{P}_{i}$ are nonempty. By Observation 1 , the set $\mathcal{S}_{i}$ is locally indistinguishable in the bipartite system $\left(\otimes_{j \in U} \mathcal{H}_{j}\right) \otimes\left(\otimes_{j \in V} \mathcal{H}_{j}\right)$. However, $\mathcal{S}_{i} \subseteq \mathcal{S}$ implies that $\mathcal{S}$ must be locally indistinguishable as bipartite states $\left(\otimes_{j \in U} \mathcal{H}_{j}\right) \otimes\left(\otimes_{j \in V} \mathcal{H}_{j}\right)$. Hence we obtain a contradiction. So the set $\mathcal{S}$ must be genuinely nonlocal.

\section{CONCLUSION AND DISCUSSION}

We study a strong form of locally indistinguishable set of fully product states called genuinely nonlocal set. We generalize the results of locally indistinguishable product states in bipartite system in Ref. [42] but provide a much more elegant proof. Based on a simple observation, we extend the results of Zhang et al. in Ref. [37] to the cases of genuinely nonlocal sets. Moreover, we extend these results to a much more general setting by relating the construction of genuinely nonlocal sets with the connectivity of some graphs. As a consequence, we can show that there always exists some genuinely nonlocal set of fully product states in $\otimes_{i=1}^{L} \mathbb{C}^{d_{i}}$ provided $d_{i} \geq 3$ for all $i$. One should note that the genuinely nonlocal set we constructed here maybe locally reducible under the concept introduced in Ref. [52]. Therefore, it is interesting to find some method to characterize the locally irreducible settings. 
Acknowledgments This work is supported by National Natural Science Foundation of China (11771419, 11875160, 11901084, 12005092, and U1801661), the China Postdoctoral Science Foundation (2020M681996), the Natural Science Foundation of Guang-dong Province (2017B030308003), the Key R\&D Program of Guangdong province (2018B030326001), the Guang-dong Innovative and Entrepreneurial Research TeamProgram (2016ZT06D348), the Science, Technology and
Innovation Commission of Shenzhen Municipality (JCYJ20170412152620376 and JCYJ20170817105046702 and KYTDPT20181011104202253), the Economy, Trade and Information Commission of Shenzhen Municipality (201901161512), the Research startup funds of DGUT (GC300501-103), the Fundamental Research Funds for the Central Universities, and Anhui Initiative in Quantum Information Technologies under Grant No. AHY150200.
[1] M. A. Nielsen and I. L. Chuang. Quantum Computation and Quantum Information(Cambridge University Press, Cambridge, U.K., 2004).

[2] C. H. Bennett, D. P. DiVincenzo, C. A. Fuchs, T. Mor, E. Rains, P. W. Shor, J. A. Smolin, and W. K. Wootters. Quantum nonlocality without entanglement. Phys. Rev. A 59, 1070 (1999).

[3] B. M. Terhal, D. P. DiVincenzo, and D. W. Leung. Hiding Bits in Bell States. Phys. Rev. Lett. 86, 5807 (2001).

[4] D. P. DiVincenzo, D.W. Leung and B.M. Terhal. Quantum data hiding. IEEE Trans. Inf. Theory 48, 580 (2002).

[5] D. Markham and B. C. Sanders. Graph States for Quantum Secret Sharing. Phys. Rev. A 78, 042309 (2008).

[6] R. Rahaman and M. G. Parker. Quantum scheme for secret sharing based on local distinguishability. Phys. Rev. A 91, 022330 (2015).

[7] J. Wang, L. Li, H. Peng, and Y. Yang. Quantum-secretsharing scheme based on local distinguishability of orthogonal multiqudit entangled states. Phys. Rev. A 95, 022320 (2017).

[8] J. Walgate and L. Hardy. Nonlocality Asymmetry and Distinguishing Bipartite States. Phys. Rev. Lett. 89, 147901 (2002).

[9] J. Walgate, A. J. Short, L. Hardy, and V. Vedral. Local Distinguishability of Multipartite Orthogonal Quantum States. Phys. Rev. Lett. 85, 4972 (2000).

[10] S. Ghosh, G. Kar, A. Roy, A. Sen(De), and U. Sen. Distinguishability of Bell States. Phys. Rev. Lett. 87, 277902 (2001).

[11] H. Fan. Distinguishability and Indistinguishability by Local Operations and Classical Communication. Phys. Rev. Lett. 92, 177905 (2004).

[12] M. Nathanson. Distinguishing bipartitite orthogonal states using LOCC: Best and worst cases. J. Math. Phys. (N.Y.) 46, 062103 (2005).

[13] H. Fan. Distinguishing bipartite states by local operations and classical communication. Phys. Rev. A 75, 014305 (2007).

[14] S. M. Cohen. Local distinguishability with preservation of entanglement. Phys. Rev. A 75, 052313 (2007).

[15] S. Bandyopadhyay, S. Ghosh, and G. Kar. LOCC distinguishability of unilaterally transformable quantum states.
New J. Phys. 13123013 (2011).

[16] N. Yu, R. Duan, and M. Ying. Four Locally Indistinguishable Ququad-Ququad Orthogonal Maximally Entangled States. Phys. Rev. Lett. 109, 020506 (2012).

[17] A. Cosentino. Positive partial transpose indistinguishable states via semidefinite programming. Phys. Rev. A 87, 012321 (2013).

[18] M.-S. Li, Y.-L. Wang, S.-M. Fei and Z.-J. Zheng. $d$ locally indistinguishable maximally entangled states in $\mathbb{C}^{d} \otimes \mathbb{C}^{d}$. Phys. Rev. A 91, 042318 (2015).

[19] S. X. Yu and C. H. Oh, Detecting the local indistinguishability of maximally entangled states. arXiv:1502.01274v1.

[20] Y.-L. Wang, M.-S. Li, and Z.-X. Xiong. One-way local distinguishability of generalized Bell states in arbitrary dimension. Phys. Rev. A 99, 022307 (2019).

[21] Z.-X. Xiong, M.-S. Li, Z.-J. Zheng, C.-J. Zhu, and S.M. Fei. Positive-partial-transpose distinguishability for lattice-type maximally entangled states. Phys. Rev. A 99, 032346 (2019).

[22] M.-S. Li, S.-M. Fei, Z.-X. Xiong, and Y.-L. Wang. Twistteleportation-based local discrimination of maximally entangled states. SCIENCE CHINA Physics, Mechnics \& Astronomy 63 8, 280312 (2020).

[23] S. De Rinaldis. Distinguishability of complete and unextendible product bases. Phys. Rev. A 70, 022309 (2004).

[24] M. Horodecki, A. Sen(De), U. Sen, and K. Horodecki. Local Indistinguishability: More Nonlocality with Less Entanglement. Phys. Rev. Lett. 90, 047902 (2003).

[25] C. H. Bennett, D. P. DiVincenzo, T. Mor, P. W. Shor, J. A. Smolin, and B. M. Terhal. Unextendible Product Bases and Bound Entanglement. Phys. Rev. Lett. 82, 5385 (1999).

[26] D. P. DiVincenzo, T. Mor, P. W. Shor, J. A. Smolin, and B. M. Terhal. Unextendible product bases, uncompletable product bases and bound entanglement. Comm. Math. Phys. 238, 379 (2003).

[27] Z.-C. Zhang, F. Gao, G.-J. Tian, T.-Q. Cao and Q.-Y. Wen. Nonlocality of orthogonal product basis quantum states. Phys. Rev. A 90, 022313 (2014).

[28] Z.-C. Zhang, F. Gao, S.-J. Qin, Y.-H. Yang, and Q.Y. Wen. Nonlocality of orthogonal product states. Phys. Rev. A 92, 012332 (2015). 
[29] Z.-C. Zhang, F. Gao, Y. Cao, S.-J. Qin, and Q.-Y. Wen. Local indistinguishability of orthogonal product states. Phys. Rev. A 93, 012314 (2016).

[30] G.-B. Xu, Y.-H. Yang, Q.-Y. Wen, S.-J. Qin, and F. Gao. Locally indistinguishable orthogonal product bases in arbitrary bipartite quantum system. Sci. Rep. 6, 31048 (2016).

[31] G.-B. Xu, Q.-Y. Wen, S.-J. Qin, Y.-H. Yang, and F. Gao. Quantum nonlocality of multipartite orthogonal product states. Phys. Rev. A 93, 032341 (2016).

[32] X.-Q. Zhang, X.-Q. Tan, J. Weng, and Y.-J. Li. LOCC indistinguishable orthogonal product quantum states. Sci. Rep. 6, 28864 (2016).

[33] Y.-L. Wang, M.-S. Li, Z.-J. Zheng, and S.-M. Fei. Nonlocality of orthogonal product-basis quantum states. Phys. Rev. A 92, 032313 (2015).

[34] Y.-L. Wang, M.-S. Li, Z.-J. Zheng, and S.-M. Fei. The local indistinguishability of multipartite product states. Quant. Info. Proc. 16, 5 (2017).

[35] Y. Feng and Y.-Y. Shi. Characterizing Locally Indistinguishable Orthogonal Product States. IEEE Trans. Inf. Theory 55, 2799 (2009).

[36] Y.-H. Yang, F. Gao, G.-J. Tian, T.-Q. Cao, and Q.Y. Wen. Local distinguishability of orthogonal quantum states in a $2 \otimes 2 \otimes 2$ system. Phys. Rev. A 88, 024301 (2013).

[37] Z.-C. Zhang, K.-J. Zhang, F. Gao, Q.-Y. Wen, and C. $\mathrm{H}$. Oh. Construction of nonlocal multipartite quantum states. Phys. Rev. A 95, 052344 (2017).

[38] X. Zhang, J. Weng, X. Tan, and W. Luo. Indistinguishability of pure orthogonal product states by LOCC. Quantum Inf. Process. 16, 168 (2017).

[39] S. Halder. Several nonlocal sets of multipartite pure orthogonal product states. Phys. Rev. A 98, 022303 (2018).

[40] M.-S. Li and Y.-L. Wang. Alternative method for deriving nonlocal multipartite product states. Phys. Rev. A 98, 052352 (2018).

[41] S. Halder, and C. Srivastava. Locally distinguishing quantum states with limited classical communication. Phys. Rev. A 101, 052313 (2020).

[42] G.-B. Xu, and D.-H. Jiang. Novel methods to construct nonlocal sets of orthogonal product states in any bipartite high-dimensional system. arXiv:2003.08291v1.

[43] D.-H. Jiang, and G.-B. Xu. Nonlocal sets of orthogonal product states in arbitrary multipartite quantum system.
Phys. Rev. A 102, 032211 (2020).

[44] S. M. Cohen. Understanding entanglement as resource: Locally distinguishing unextendible product bases. Phys. Rev. A 77, 012304 (2008).

[45] S. Bandyopadhyay, S. Halder, and M. Nathanson. Entanglement as a resource for local state discrimination in multipartite systems. Phys. Rev. A 94, 022311 (2016).

[46] Z.-C. Zhang, F. Gao, T.-Q. Cao, S.-J. Qin, and Q.-Y. Wen. Entanglement as a resource to distinguish orthogonal product states. Sci. Rep. 6, 30493 (2016).

[47] S. Bandyopadhyay, S. Halder, and M. Nathanson. Optimal resource states for local state discrimination. Phys. Rev. A 97, 022314 (2018).

[48] L.-J. Li, F. Gao, Z.-C. Zhang, and Q.-Y. Wen. Local distinguishability of orthogonal quantum states with no more than one ebit of entanglement. Phys. Rev. A 99, 012343 (2019).

[49] K. Feng. Unextendible product bases and 1-factorization of complete graphs. Disc. App. Math. 154, 942 (2006).

[50] N. Johnston. The structure of qubit unextendible product bases. J. Phys. A: Math. Theor. 47, 424034 (2014).

[51] Jianxin Chen, and N. Johnston. The Minimum Size of Unextendible Product Bases in the Bipartite Case (and Some Multipartite Cases). Commun. Math. Phys. 333, 351-365 (2015).

[52] S. Halder, M. Banik, S. Agrawal, and S. Bandyopadhyay. Strong Quantum Nonlocality without Entanglement. Phys. Rev. Lett. 122, 040403 (2019).

[53] Z.-C. Zhang and X. Zhang. Strong quantum nonlocality in multipartite quantum systems. Phys. Rev. A 99, 062108 (2019).

[54] S. Rout, A.G. Maity, A. Mukherjee, S. Halder, and M. Banik. Genuinely nonlocal product bases: Classification and entanglement-assisted discrimination. Phys. Rev. A 100, 032321 (2019).

[55] S. Rout, A. G. Maity, A. Mukherjee, S. Halder, and M. Banik. Local state discrimination and ordering of multipartite entangled states. arXiv:1910.14308.

[56] P. Yuan, G. J. Tian, and X. M. Sun. Strong quantum nonlocality without entanglement in multipartite quantum systems. Phys. Rev. A 102, 042228 (2020).

[57] F. Shi, M. Hu, L. Chen, and X. Zhang. Strong quantum nonlocality with entanglement. Phys. Rev. A 102, 042202 (2020). 